\title{
Dynamic Tariff-Subsidy Method for PV and V2G Congestion Management in Distribution Networks
}

\author{
Huang, Shaojun; Wu, Qiuwei
}

Published in:

IEEE Transactions on Smart Grid

Link to article, DOI:

10.1109/TSG.2019.2892302

Publication date:

2019

Document Version

Peer reviewed version

Link back to DTU Orbit

Citation (APA):

Huang, S., \& Wu, Q. (2019). Dynamic Tariff-Subsidy Method for PV and V2G Congestion Management in Distribution Networks. IEEE Transactions on Smart Grid, 10(5), 5851-5860.

https://doi.org/10.1109/TSG.2019.2892302

\section{General rights}

Copyright and moral rights for the publications made accessible in the public portal are retained by the authors and/or other copyright owners and it is a condition of accessing publications that users recognise and abide by the legal requirements associated with these rights.

- Users may download and print one copy of any publication from the public portal for the purpose of private study or research.

- You may not further distribute the material or use it for any profit-making activity or commercial gain

- You may freely distribute the URL identifying the publication in the public portal

If you believe that this document breaches copyright please contact us providing details, and we will remove access to the work immediately and investigate your claim. 


\title{
Dynamic Tariff-Subsidy Method for PV and V2G Congestion Management in Distribution Networks
}

\author{
Shaojun Huang, Senior Member, IEEE, and Qiuwei Wu, Senior Member, IEEE
}

\begin{abstract}
This paper proposes a dynamic tariff-subsidy (DTS) method for congestion management in distribution networks with high penetration of photovoltaics (PV), heat pumps (HPs) and electric vehicles (EVs) with vehicle-to-grid (V2G) function. The DTS method is an extension of the dynamic tariff method proposed in the previous study. With the DTS, the regulation prices can be positive (tariff) or negative (subsidy). The study shows that the negative regulation price is necessary and very effective to solve congestion due to feed-in power flows, such as PVs and EVs in the V2G mode. In the study, dual decomposition of a convex quadratic model is proposed in addition to a conventional method for the DTS calculation. The case studies on the Roy Billinton Test System (RBTS) demonstrate the efficacy of the DTS method for congestion management in distribution networks.
\end{abstract}

Index Terms-- Congestion management, distribution system operator (DSO), dynamic tariff-subsidy, electric vehicle (EV), heat pump (HP), photovoltaics (PV).

\section{NOMENCLATURE}

Sets

$N_{B} \quad$ set of aggregators

$N_{T} \quad$ set of planning periods

$N_{L} \quad$ set of lines

$N_{d} \quad$ set of demand bus

\section{Parameters}

$A_{i, t, t} \quad$ coefficient matrix, describe the relations between the power consumption and temperature change of the household $\left({ }^{\circ} \mathrm{C} / \mathrm{kW}\right)$

$B \quad$ power tariff in matrix form $(\mathrm{DKK} / \mathrm{kWh} / \mathrm{kWh})$

$D \quad$ power transfer distribution factor (PTDF)

$E_{i} \quad$ customer to load bus mapping matrix

$F_{t} \quad$ line loading limit of active power $(\mathrm{kW})$

$K_{i, t}^{a, \min } \quad$ lower temperature limit $\left({ }^{\circ} \mathrm{C}\right)$

$K_{i, t}^{a, \max } \quad$ upper temperature limit $\left({ }^{\circ} \mathrm{C}\right)$

Corresponding author: Qiuwei Wu (email: qw@elektro.dtu.dk).

S. Huang is with the Center for Energy Informatics, Mærsk Mc-Kinney Møller Institute, University of Southern Denmark (SDU), 5230 Odense M, Denmark (e-mail: shahu@mmmi.sdu.dk).

Q. Wu is with the Center for Electric Power and Energy (CEE), Department of Electrical Engineering, Technical University of Denmark (DTU), 2800 Kgs. Lyngby, Denmark and Harvard China Project, School of Engineering and Applied Sciences, Harvard University, 29 Oxford Street, Cambridge, MA 02138, USA (e-mail: qw@elektro.dtu.dk, qiuwu@seas.harvard.edu).

\begin{tabular}{|c|c|}
\hline$\underline{V}, \bar{V}$ & voltage lower/upper limit (p.u.) \\
\hline$V_{0}$ & voltage at node 0 (p.u.) \\
\hline$Y_{L L}$ & $\begin{array}{l}\text { the matrix obtained by removing the first row and } \\
\text { column of the nodal admittance matrix }(\mathrm{S})\end{array}$ \\
\hline$Z$ & the inverse matrix of $Y_{L L}(\mathrm{ohm})$ \\
\hline$C_{t}$ & forecast baseline energy price $(\mathrm{DKK} / \mathrm{kWh})$ \\
\hline$d_{i, t}$ & discharging power of EVs due to driving $(\mathrm{kW})$ \\
\hline$e_{i}^{\min }$ & $\begin{array}{l}\text { lower limit of the state of charge (SOC) level } \\
(\mathrm{kWh})\end{array}$ \\
\hline$e_{i}^{\max }$ & upper limit of the SOC level (kWh) \\
\hline$e_{i, 0}$ & initial SOC level (kWh) \\
\hline$p_{i, t}^{\max }$ & discharging/charging power limit of EVs $(\mathrm{kW})$ \\
\hline$\hat{p}_{i}^{\max }$ & power limit of HPs $(\mathrm{kW})$ \\
\hline$p_{t}^{c}$ & active conventional power at each load point $(\mathrm{kW})$ \\
\hline$p_{t}^{p v}$ & $\mathrm{PV}$ active power $(\mathrm{kW})$ \\
\hline$q_{t}^{c}$ & reactive power at each load point/bus (kVar) \\
\hline$u_{i, t}$ & constant vector \\
\hline$\alpha$ & step size \\
\hline$\Delta t$ & planning period ( 1 hour in this paper) \\
\hline \multicolumn{2}{|c|}{ Vector Variables } \\
\hline$p_{i, t}$ & $\begin{array}{l}\text { charging/discharging power of EVs of one aggre- } \\
\text { gator }(\mathrm{kW})\end{array}$ \\
\hline$\hat{p}_{i, t}$ & power of HPs of one aggregator $(\mathrm{kW})$ \\
\hline$r_{t}$ & regulation price, i.e., DTS rates (DKK/kWh) \\
\hline$S_{t}$ & $\begin{array}{l}\text { total apparent power (complex variable) at each } \\
\text { load point (kVA) }\end{array}$ \\
\hline$\lambda_{t}^{-}, \lambda_{t}^{+}$ & Lagrange multiplier (LM) of line limit constraint \\
\hline$\omega_{t}^{-}, \omega_{t}^{+}$ & LM of voltage constraint \\
\hline
\end{tabular}

Other Symbols

$n_{*} \quad$ cardinality of $N_{*}$, i.e. $n_{*}=\left|N_{*}\right|$

*T transpose of matrix *

* element-wise conjugate of complex vector/matrix

\section{INTRODUCTION}

GUTURE power systems and electricity markets are ex1 pected to be largely influenced by renewable energy, which provides higher and higher share of the total energy 
production. Intermittent renewable energy production, such as wind and photovoltaics (PV) power, can cause significant balance issues to the power system. Also, electricity prices fluctuate a lot due to intermittent renewable energy production in the system. The price is not only a signal that reflects the balance of the production and consumption of the systems, but also a means that can influence the production and consumption profiles.

Nowadays, distribution networks have gained more and more attention because a large number of distributed energy resources (DERs), such as electric vehicles (EVs), heat pumps (HPs) and PVs, are integrated into them. DERs can support the power systems in many ways. However, the potential congestion in distribution networks due to DERs must be handled. In previous studies, a number of coordination methods have been proposed to solve the congestion problems. Such methods include direct control methods and indirect control methods or market-based methods [1]. Direct control methods, e.g., reconfiguration [2], direct active power control [3] and direct reactive control [4], do not consider the individual needs of the customers. However, these methods do consider the overall social welfare and are suitable for countries where there are no energy markets. Indirect control methods include both pricebased methods and incentive-based methods, e.g., the dynamic tariff (DT) [5]-[8], distribution locational marginal price (DLMP) [9], [10], line shadow price method [11], subsidybased method [12]-[15], multi-agent system method [16], [17], decentralized protocol [18], [19] and probabilistic congestion management method [20]-[23]. Indirect control methods have customers or aggregators actively involved in the congestion management process and maximize the individual profit (or minimize the individual cost). In the DT method, the individual benefits are in line with the overall benefits. It is also beneficial to combine several of the abovementioned methods.

Most of the abovementioned methods do not consider the PV or PV plus vehicle-to-grid (V2G) cases. There are studies in the literature focusing on PV and V2G. In [24], workplace EVs were employed to consume local PV production to reduce the charging cost. Meanwhile, the V2G function and ancillary service market are considered to maximize the profit. In [25], a probabilistic congestion management method was proposed to manage PVs and EVs including the V2G function. In [26], a coordination strategy to coordinate EVs and PVs was proposed to mitigate PV production uncertainties. However, all these methods are based on direct control; they either do not consider prices, or they function as price takers and pricing strategies are not considered.

In this paper, market-based methods are employed for congestion management in distribution networks with high penetration of PVs, HPs and V2G enabled EVs. This paper extends the DT method to handle congestion management caused by $\mathrm{PV}$ and V2G. The main contributions of this paper are summarized as follows: 1) Propose a dynamic tariff-subsidy (DTS) method for congestion management in distribution networks. In the DTS method, the regulation price can be negative. A negative regulation price can be considered as a subsidy for the owners/aggregators of DERs, and it can effectively solve congestion problems due to feed-in power flows from PVs or EVs in the V2G mode (overloading in the upward direction). A comparison between the DTS method and the previous DT method and dynamic subsidy method [15] is shown in Table I; 2) Formulate two models for the DTS calculation. One model employs a single optimization based on forecasted parameters of DERs. The other model has an iterative process based on the dual decomposition. The two models obtain the same optimal planning if all the parameters are the same.

TABLE I

COMPARISON BETWEEN DIFFERENT METHODS

\begin{tabular}{|c|c|c|c|}
\hline & $\begin{array}{l}\text { Dynamic } \\
\text { Tariff }\end{array}$ & $\begin{array}{l}\text { Dynamic Subsi- } \\
\text { dy }\end{array}$ & $\begin{array}{l}\text { mic Tariff- } \\
\text { dy }\end{array}$ \\
\hline $\begin{array}{l}\text { Price } \\
\text { whic }\end{array}$ & $\begin{array}{l}\text { Congestion } \\
\text { hour }\end{array}$ & $\begin{array}{l}\text { Non-congestion } \\
\text { hour }\end{array}$ & $\mathrm{Co}$ \\
\hline $\begin{array}{l}\text { Congestion } \\
\text { type }\end{array}$ & $\begin{array}{l}\text { Overloading } \\
\text { downward }\end{array}$ & $\begin{array}{l}\text { Overloading } \\
\text { downward }\end{array}$ & $\begin{array}{l}\text { Both downward } \\
\text { and upward }\end{array}$ \\
\hline
\end{tabular}

The rest of the paper is organized as follows. The market structure and DTS concept are presented in Section III. The DTS calculation by a single optimization method is presented in Section IV. The equivalence between the single optimization and distributed aggregator optimization is also discussed in this section. Then, a dual-decomposition-based method for the DTS calculation is described in Section V. In Section VI, case studies are presented and discussed, followed by conclusions.

\section{MARKET STRUCTURE AND DTS}

In this section, the market basis and mechanism of the DTS method for congestion management in distribution networks is presented.

\section{A. The Underlying Markets}

Similar to the DT method, the DTS method works along with a day-ahead electricity market. The day-ahead market settles the bids of the retailers/aggregators and producers. The bids are settled for the next day with an hourly base (or smaller periods). The Nordic day-ahead spot market is an example of such markets.

Traditionally, retailers forecast the consumption profile for the next day and submit bids to the day-ahead market according to the forecast. Nowadays, as more and more renewable energy and flexible demands, such as EVs and HPs, enter the market, forecasting and making optimal energy plans are challenging for aggregators. Aggregators are replacing retailers due to their new roles: buying and/or selling energy for DERs, including EVs, HPs, PVs, etc.

Another challenge is that the day-ahead electricity market is at the transmission network level and there is no mature market at the distribution network level yet. As a significant share of the DERs is connected to distribution networks, especially the PVs, potential congestion must be properly handled when the penetration level is high. Aggregators must coordinate with the DSO for congestion management when they submit their bids to the day-ahead market. In this paper, the 
DTS method is proposed for such coordination. It is based on the market mechanism explained in the following subsection.

\section{B. The DTS Method}

The DT method proposed in [7] can manage congestion problems caused by EVs and HPs, i.e., flexible demands only. In order to manage congestion problems caused by PVs and EVs (V2G), i.e., feed-in power flows, the DTS contains both positive and negative rates. The negative rates can be considered as subsidies as the name 'DTS' indicates.

The DTS rates are determined by the DSO before the dayahead market. Therefore, when the aggregators make optimal energy planning for the day-ahead market, they are expected to consider the DTS together with the forecast of day-ahead market prices. The DTS is a distribution network regulation price. It is determined in a way such that the aggregators' energy planning based on DTS is expected not to cause congestion in the distribution network.

In addition to the ability to manage congestion, another important feature of the DTS method is that it leads to an overall minimized cost (maximized social welfare) of the whole distribution network, as the DT method does [7]. This feature is kept during the extension from the DT to the DTS. The key is to prove that the overall optimization at the DSO side (minimize the total cost or maximize the total social welfare) respecting the network constraints is equivalent to the distributed optimizations at the aggregators side. Without this, a price-based congestion management will not lead to the overall maximized social welfare, even though each individual optimization of the aggregators is achieved.

As shown in [7], the quadratic program based on sensitivity is the key for the success of the proof of equivalence. In [27], it is pointed out that the power tariff can substitute the sensitivity to formulate quadratic programs for the DSO and aggregators optimizations. In this paper, a small fixed power tariff (FPT) is employed to formulate quadratic programs (see detailed formulation in Section IV.A). Hence, the aggregators will make their optimal planning based on the FTP (fixed rate for a month or a season) and the received DTS daily from the DSO.

\section{Procedure of The DTS Method}

The procedure of the DTS method for congestion management has two options. The difference between the two options is the DTS calculation method. The first option is to calculate DTS rates by a single optimization at the DSO side, which is similar to the DT method [7]. The second option is to calculate the DTS rates by an iteration process with back and forth interactions between the DSO and aggregators, like the distributed DT method [28].

The detailed procedure for the first option is illustrated in Fig. 1. The DSO obtains necessary information for the DTS calculation. The aggregators report information of DERs to the DSO (an optional step). The DSO calculates the DTS and sends them to the aggregators. The aggregators make optimal planning based on the power tariff, forecast energy prices and DTS. The aggregators submit bids to the day-ahead market. The aggregators report the final schedules in an aggregated form to the DSO.

The detailed procedure for the second option is illustrated in Fig. 2. The DSO obtains necessary information for the DTS calculation. The DSO sends initial DTS (all zeros) to the aggregators. The aggregators make optimal planning based on the forecasted energy price, power tariff and DTS. The aggregators report the schedules (an aggregated form) to the DSO. Then repeat this process until there is no congestion. The DSO obtains the final DTS. The aggregators submit the bids to the day-ahead market.

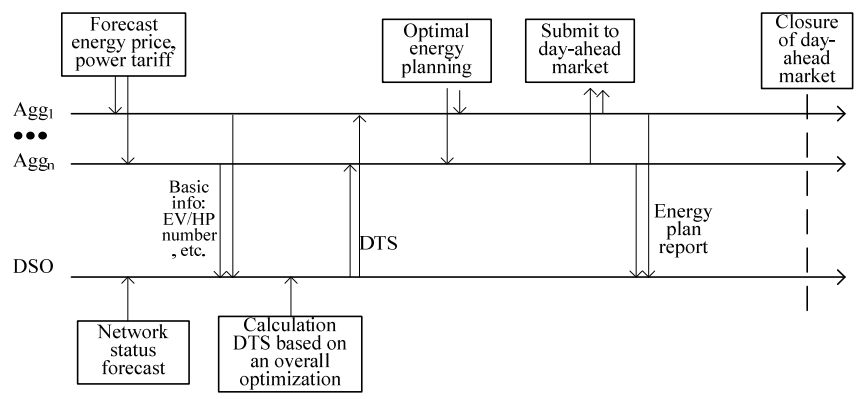

Fig. 1. Illustration of the DTS method with a single optimization process

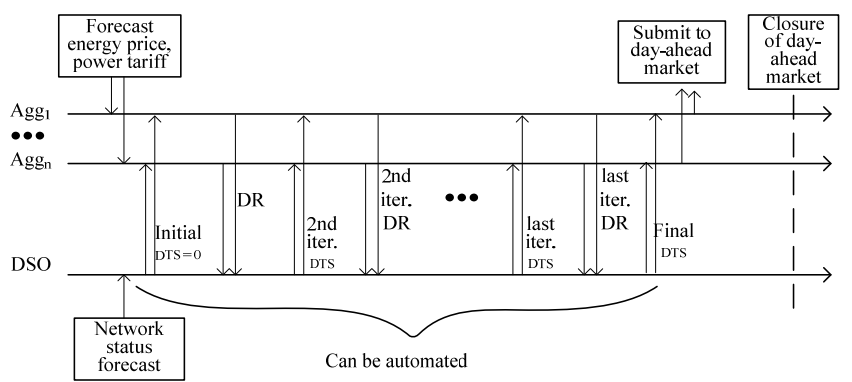

Fig. 2. Illustration of the DTS method with an iterative process

The advantage of the first option is its simplicity but may not be able to solve congestion accurately. The second option is more complicate but with higher accuracy. Details of the two options are presented in Sections IV and V, respectively.

\section{DTS CALCULATION}

\section{A. Modelling of DERs in Distribution Networks}

The modelling of HPs is the same as in [7] (see Appendix C). For brevity, the model of the optimal planning of HPs is directly given as,

$$
\min _{\hat{p}_{i, t}} \sum_{i \in N_{B}, t \in N_{T}}\left(\hat{p}_{i, t}^{T} B \hat{p}_{i, t}+\left(c_{t} \mathbf{1}\right)^{T} \hat{p}_{i, t}\right)
$$

subject to,

$$
\begin{gathered}
K_{i, t}^{a, \text { min }} \leq \sum_{t_{-} \leq t} A_{i, t, t_{-}} \hat{p}_{i, t_{-}}+u_{i, t} \leq K_{i, t}^{a, \max }, \quad i \in N_{B}, \forall t \in N_{T}, \\
0 \leq \hat{p}_{i, t} \leq \hat{p}_{i, t}^{\max }, \quad i \in N_{B}, t \in N_{T} .
\end{gathered}
$$

Constraint (2) represents the thermal limits of households, where coefficient $A_{i, t, t_{-}}$and constant $u_{i, t}$ relate the power to the household inside temperature (the method to determine $A$ and $u$ can be seen in Appendix C), and parameters $K_{i, t}^{a, \min }$ and $K_{i, t}^{a, \max }$ are the temperature limit settings of each house- 
hold. Constraint (3) gives the input power limits of HPs. The HP power variable $\hat{p}_{i, t}$ is always positive, representing power consumption. In the objective function, parameter $B$ (a diagonal matrix with identical elements equal to the power tariff) represents the constant power tariff rate, fixed for a month or a season. The power tariff is introduced to smooth the energy planning; moreover, the quadratic terms can avoid multiple solutions which compromises the DR effect of the aggregators [7] (see Appendix B for further explanation). The total power tariff cost is proportional to the square of the HP power $\hat{p}_{i, t}$.

Parameter $c_{t}$ represents the forecasted day-ahead energy price for each hour.

The PV production is modelled as negative power, $p_{i, t}^{p v}$, representing power production. Its reactive power is neglected.

The EVs are modelled as follows.

$$
\min _{p_{i, t}} \sum_{i \in N_{B}, t \in N_{T}}\left(p_{i, t}{ }^{T} B p_{i, t}+\left(c_{t} \mathbf{1}\right)^{T} p_{i, t}\right),
$$

subject to,

$$
\begin{gathered}
e_{i}^{\min } \leq \Delta t \cdot \sum_{t_{-} \leq t}\left(p_{i, t_{-}}-d_{i, t_{-}}\right)+e_{i 0} \leq e_{i}^{\max }, \quad i \in N_{B}, \forall t \in N_{t}, \\
\text { alternatively, } \sum_{t}\left(p_{i, t}-d_{i, t}\right)=0, \quad i \in N_{B}, \\
\quad-p_{i, t}^{\max } \leq p_{i, t} \leq p_{i, t}^{\max }, \quad i \in N_{B}, \forall t \in N_{T} .
\end{gathered}
$$

The EV power variable, $p_{i, t}$, is either positive $(\mathrm{G} 2 \mathrm{~V}$, charging) or negative (V2G, discharging). Constraint (5) limits the SOC of the EV battery within the allowed range, e.g., $10 \% \sim 90 \%$ of the maximum SOC level. Alternatively, constraint (6) can be used to achieve a similar goal. With (6), it is to balance the total charging/discharging and the total consumption due to driving $\left(d_{i, t}\right)$; therefore, the SOC at the end of the whole planning period (24 hours according to the dayahead market) is kept the same as the initial SOC. Which one to be employed depends on the agreement between the aggregators and their customers. In this paper, (5) is chosen. Since both are linear constraints, the choice will not affect the analysis in the rest of the paper.

\section{B. DTS Calculation by a Single Optimization}

The DSO is responsible for the DTS calculation. It is assumed in this subsection that the DSO has obtained all the parameters of HPs, EVs and PVs by forecast.

Putting all the DER models together, adding the network constraints, the optimization at the DSO side for the DTS calculation is obtained as,

\section{Optimization I:}

$$
\begin{array}{r}
\min _{p_{i, t}, \hat{p}_{i, t}} \sum_{i \in N_{B}, t \in N_{T}}\left(p_{i, t}{ }^{T} B p_{i, t}+\left(c_{t} \mathbf{1}\right)^{T} p_{i, t}\right. \\
\left.+\hat{p}_{i, t}{ }^{T} B \hat{p}_{i, t}+\left(c_{t} \mathbf{1}\right)^{T} \hat{p}_{i, t}\right)
\end{array},
$$

subject to (2), (3), (5), (7) and,

$$
\begin{gathered}
-F_{t} \leq D \operatorname{Re}\left(s_{t}\right) \leq F_{t}, \forall t \in N_{T},\left(\lambda_{t}^{-}, \lambda_{t}^{+}\right), \\
\frac{\underline{V}}{V_{0}} \leq 1-\frac{1}{V_{0}^{2}} \operatorname{Re}\left(Z s_{t}\right) \leq \frac{\bar{V}}{V_{0}}, \forall t \in N_{T},\left(\omega_{t}^{-}, \omega_{t}^{+}\right),
\end{gathered}
$$

$$
\begin{gathered}
\operatorname{Re}\left(s_{t}\right)=p_{t}^{c}+\sum_{i \in N_{B}} E_{i}\left(p_{i, t}^{p v}+p_{i, t}+\hat{p}_{i, t}\right), \forall t \in N_{T}, \\
\operatorname{Im}\left(s_{t}\right)=q_{t}^{c}, \forall t \in N_{T} .
\end{gathered}
$$

Constraints (9) and (10) are to compare the power flow and voltage level with the network limits, i.e., line flow and voltage limits, respectively. DC power flow is employed in this paper to determine the line flows from the nodal power. Parameter $q_{t}^{c}$, representing the total reactive power at each bus, including the conventional demands, HPs and compensators, is employed to estimate the voltages. As the DTS is only for active power, reactive power is not part of the planning variables in this work. The method to estimate an approximate voltage level for each bus was proposed by [29]. The maximum errors of line flow and voltage were analyzed in [15], [29], respectively. With careful selection of the line flow and voltage limits and careful analysis of the optimization results, DCOPF should be sufficient for the DTS method.

Lagrange multipliers $\lambda_{t}^{-}, \lambda_{t}^{+}, \omega_{t}^{-}, \omega_{t}^{+}(-$represents the left inequality; + represents the right) in (9)-(10) will be used to calculate the DTS. Matrix $Z$ is the inverse matrix of the partial nodal admittance matrix $Y_{L L}$, which is a submatrix of the full admittance matrix ( $Y_{00}$ represents the slack bus),

$$
Y=\left[\begin{array}{ll}
Y_{00} & Y_{0 L} \\
Y_{L 0} & Y_{L L}
\end{array}\right] .
$$

After solving the DSO side optimization, the optimal solution is obtained as $\left(p_{i, t}{ }^{*}, \hat{p}_{i, t}{ }^{*}, s_{t}^{*}, \lambda_{t}^{-*}, \lambda_{t}^{+^{+*}}, \omega_{t}^{-{ }^{*}}, \omega_{t}^{+*}\right)$. Then, the DTS, $r_{t}$, can be determined by,

$$
r_{t}=D^{T}\left(\lambda_{t}^{+*}-\lambda_{t}^{-*}\right)-\frac{\operatorname{Re}\left(Z^{T}\right)}{V_{0}^{2}}\left(\omega_{t}^{+*}-\omega_{t}^{-*}\right) .
$$

The DTS determined by (13) has two parts: one is related to line flow constraints and the other is related to voltage constraints. If the negative line flow constraint (left inequality of (9)) is binding, the corresponding $\lambda_{t}^{+^{*}}$ is zero while $\lambda_{t}^{-*}$ is positive. This means that the DTS tends to be negative when the feed-in power, such as the PV generation, is strong and hits the line flow limit. A negative DTS is a subsidy, which can attract EVs to charge their batteries, and by doing this, the congestion due to strong feed-in power is mitigated. A similar principle applies to the voltage congestion. If voltage hits the upper limit, meaning too much feed-in power, $\omega_{t}^{+*}-\omega_{t}^{-*}$ will be positive and the DTS tends to be negative as well.

\section{Aggregator Side Optimization and Equivalence}

The DSO sends $r_{t}$ to all the aggregators. An aggregator, $i$, can map the received DTS, which is the regulation price per bus per hour, to the regulation price per household per hour by $E_{i}^{T} r_{t}$. Then use the following optimization to make optimal planning for the households that it has contracts with. For aggregator $i$,

$$
\begin{gathered}
\min _{p_{i, t}, \hat{p}_{i, t}} \sum_{t \in N_{T}}\left(p_{i, t}{ }^{T} B p_{i, t}+\left(c_{t} \mathbf{1}+E_{i}^{T} r_{t}\right)^{T} p_{i, t}\right. \\
\left.\hat{p}_{i, t}{ }^{T} B \hat{p}_{i, t}+\left(c_{t} \mathbf{1}+E_{i}^{T} r_{t}\right)^{T} \hat{p}_{i, t}\right)
\end{gathered},
$$


subject to,

$$
\begin{gathered}
K_{i, t}^{a, \min } \leq \sum_{t_{-} \leq t} A_{i, t, t_{-}} \hat{p}_{i, t_{-}}+u_{i, t} \leq K_{i, t}^{a, \max }, \quad \forall t \in N_{T}, \\
0 \leq \hat{p}_{i, t} \leq \hat{p}_{i, t}^{\max }, \quad t \in N_{T} . \\
e_{i}^{\min } \leq \Delta t \cdot \sum_{t_{-} \leq t}\left(p_{i, t_{-}}-d_{i, t_{-}}\right)+e_{i 0} \leq e_{i}^{\max }, \quad \forall t \in N_{t}, \\
-p_{i, t}^{\max } \leq p_{i, t} \leq p_{i, t}^{\max }, \quad \forall t \in N_{T} .
\end{gathered}
$$

Notice that, for aggregator $i$, subscription $i$ in (14)-(18) is fixed, and $i \in N_{B}$ is no longer needed.

As shown in [7], equivalence means that the optimization at the DSO side has the same optimal solution $\left(p_{i, t}{ }^{*}, \hat{p}_{i, t}{ }^{*}\right)$ as for the optimization problems at the aggregator side. This can be proven by comparing the KKT conditions of these optimization problems, plus the fact that all these problems are quadratic program and each has a unique optimal solution.

It should be noted that the objective function has different values for the DSO side problem and the aggregator side problems, as the aggregator side objective function has extra cost due to the DTS. The DSO will not earn profit from the DTS, i.e., the DTS collected by the DSO will eventually be spent to benefit the customers themselves. Therefore, it can be neglected when it comes to the total social welfare. This means, by using the DTS method, the optimal planning at the aggregator side can achieve the same social welfare (the minimized total energy cost) as the DSO side optimization does.

\section{Discussions on the modelling}

The modelling of the DERs in the above subsections are mainly for the day-ahead planning. The congestion management in this paper is focusing on medium voltage networks, e.g., $10 \mathrm{kV}$ in many countries. Therefore, the aggregated power of hundreds of customers at each load point is relevant to the DSO and the aggregators, rather than the individual power of each DER. Although the above modelling is detailed down to each DER, only the aggregated power of each aggregator at each load point at the operation time is required to be the same as the planned. This modelling method has several advantages. One of them is that the aggregators can use this simplified model to do the day-ahead planning. At the operation time, when there is more accurate information about their DERs, control the DERs according to their individual characteristics and requirements, i.e., whether the HPs have thermostatic regulators or the availability and SOC of EVs. However, this paper will not go into details about the operation time control.

If uncertainty is considered, the modelling needs some changes. Take the dual decomposition formulation of the DTS method (Section V) as an example. The dual decomposition has two parts: the aggregator's optimization and the DSO calculation of DTS. If price uncertainty is considered, the aggregator's optimization model will change its objective function and turn into a stochastic program. If the uncertainty of EVs and HPs is considered, the model at the aggregators side will be a chance constrained optimization. All these happen at the aggregator side and the DSO side will not change. The aggregators are free to choose deterministic or stochastic models as they prefer. The DSO will handle the uncertainties of the tradi- tional loads and PVs (negative loads). If the uncertainties are modelled by Normal distributions, the chance constraints can first be transformed into deterministic constraints, then the dual decomposition method for calculating DTS can be used.

If uncertainty is considered for the single optimization formulation of the DTS method (Section IV.B), the method in [23] can be employed to handle this situation.

\section{DTS CALCULATION BY DUAL DECOMPOSITION}

Due to the potential forecast error of the DER parameters, the DTS calculated by a single optimization at the DSO side (Optimization I) may not be able to solve the congestion accurately. This section introduces a DTS calculation method by the dual decomposition [30], which is an iterative method. The same idea has been employed for the distributed optimization based DT method in a previous study [28]. In the current study, the model is generalized to allow feed-in power flow and $\mathrm{V} 2 \mathrm{G}$ function. Also, negative DT, i.e., a subsidy, is allowed.

Since Optimization I is a quadratic program and is strictly convex, its duality gap is zero (assume Optimization I is feasible; otherwise, the congestion is not solvable and additional means must be employed by the DSO, such as incentive-based methods or, partly solve the congestion and use incentivebased methods for the remaining congestion). The dual problem of Optimization I (only dualize the coupling constraints (9) $-(10))$ is,

$$
\begin{array}{r}
\max _{\left(\lambda_{t}^{+}, \lambda_{t}^{-}, \omega_{t}^{+}, \omega_{t}^{-}\right) \geq 0}\left\{\operatorname { m i n } _ { p _ { i , t } , \hat { p } _ { i , t } } \sum _ { i \in N _ { B } , t \in N _ { T } } \left(p_{i, t}{ }^{T} B p_{i, t}+\left(c_{t} \mathbf{1}\right)^{T} p_{i, t}+\right.\right. \\
\left.\left.\hat{p}_{i, t}{ }^{T} B \hat{p}_{i, t}+\left(c_{t} \mathbf{1}\right)^{T} \widehat{p}_{i, t}\right)\right)+\sum_{t \in N_{T}}\left(\lambda_{t}^{+}\left(D \operatorname{Re}\left(s_{t}\right)-F_{t}\right)\right. \\
-\lambda_{t}^{-}\left(D \operatorname{Re}\left(s_{t}\right)+F_{t}\right)+\omega_{t}^{+}\left(1-\frac{1}{V_{0}^{2}} \operatorname{Re}\left(Z s_{t}\right)-\frac{\bar{V}}{V_{0}}\right), \\
\left.\left.-\omega_{t}^{-}\left(1-\frac{1}{V_{0}^{2}} \operatorname{Re}\left(Z s_{t}\right)-\frac{\underline{V}}{V_{0}}\right)\right)\right\}
\end{array}
$$

subject to (2), (3), (5), (7), and, (11)-(12).

Drop $s_{t}$ and (11)-(12), the dual problem will be: (20) subject to (2), (3), (5), (7).

$$
\begin{aligned}
& \max _{\left(\lambda_{t}^{+}, \lambda_{t}^{,}, \omega_{t}^{+}, \omega_{t}^{-}\right) \geq 0}\left\{\min _{p_{i, t}, \hat{p}_{i, t}, t} \sum_{i \in N_{B}, t \in N_{T}}(\right. \\
& p_{i, t}{ }^{T} B p_{i, t}+\left(c_{t} \mathbf{1}+E_{i}^{T}\left(D^{T}\left(\lambda_{t}^{+}-\lambda_{t}^{-}\right)-\frac{\operatorname{Re}\left(Z^{T}\right)}{V_{0}^{2}}\left(\omega_{t}^{+}-\omega_{t}^{-}\right)\right)\right)^{T} p_{i, t} \\
& +\widehat{p}_{i, t}{ }^{T} B \widehat{B}_{i, t}+\left(c_{t} \mathbf{1}+E_{i}^{T}\left(D^{T}\left(\lambda_{t}^{+}-\lambda_{t}^{-}\right)-\frac{\operatorname{Re}\left(Z^{T}\right)}{V_{0}^{2}}\left(\omega_{t}^{+}-\omega_{t}^{-}\right)\right)\right)^{T} \hat{p}_{i, t} \\
& )+\sum_{t \in N_{T}}\left(\lambda_{t}^{+T}\left(D\left(p_{t}^{c}+\sum_{i \in N_{B}} E_{i} p_{i, t}^{p v}\right)-F_{t}\right)\right. \\
& -\lambda_{t}^{-T}\left(D\left(p_{t}^{c}+\sum_{i \in N_{B}} E_{i} p_{i, t}^{p v}\right)+F_{t}\right) \\
& +\omega_{t}^{+T}\left(1-\frac{1}{V_{0}^{2}}\left(\operatorname{Re}(Z)\left(p_{t}^{c}+\sum_{i \in N_{B}} E_{i} p_{i, t}^{p v}\right)+\operatorname{Im}(Z) q_{t}^{c}\right)-\frac{\bar{V}}{V_{0}}\right) \\
& \left.\left.-\omega_{t}^{-T}\left(1-\frac{1}{V_{0}^{2}}\left(\operatorname{Re}(Z)\left(p_{t}^{c}+\sum_{i \in N_{B}} E_{i} p_{i, t}^{p \nu}\right)+\operatorname{Im}(Z) q_{t}^{c}\right)-\frac{\underline{V}}{V_{0}}\right)\right)\right\}
\end{aligned}
$$

Notice that the last four rows in (20) are constants for the inner optimization and can be ignored, while the first two rows are decomposable for each aggregator. This is an expected 
result of dualizing the coupling constraints (9)-(10). The outer optimization is respect to dual variables $\left(\lambda_{t}^{+}, \lambda_{t}^{-}, \omega_{t}^{+}, \omega_{t}^{-}\right)$, and can be solved by the subgradient method [31].

The procedure to solve the dual problem is as follows.

Firstly, initial values for the dual variables $\left(\lambda_{t}^{+}, \lambda_{t}^{-}, \omega_{t}^{+}, \omega_{t}^{-}\right)$ are determined, e.g., all zeros, as an initial solution for the outer problem. Then the initial DTSs are also all zeros.

Secondly, the DTSs are sent to aggregators. As the inner problem is decomposable, each subproblem of the inner problem is solved by the corresponding aggregator using (14)-(18).

Thirdly, the aggregators report the solution to the DSO. Then the DSO solves the outer problem by the subgradient method. The subgradients are calculated according to constraints (9)-(10). Then the dual variables $\left(\lambda_{t}^{+}, \lambda_{t}^{-}, \omega_{t}^{+}, \omega_{t}^{-}\right)$are updated by a small constant step size $\alpha$ :

$$
\begin{gathered}
\left(\lambda_{t}^{+}\right)^{(k+1)}=\left(\lambda_{t}^{+}\right)^{(k)}+\alpha\left(D \operatorname{Re}\left(s_{t}^{(k)}\right)-F_{t}\right), \forall t \in N_{T}, \\
\left(\lambda_{t}^{-}\right)^{(k+1)}=\left(\lambda_{t}^{-}\right)^{(k)}+\alpha\left(-D \operatorname{Re}\left(s_{t}^{(k)}\right)-F_{t}\right), \forall t \in N_{T}, \\
\left(\omega_{t}^{+}\right)^{(k+1)}=\left(\omega_{t}^{+}\right)^{(k)}+\alpha\left(1-\frac{1}{V_{0}^{2}} \operatorname{Re}\left(Z \overline{s_{t}^{(k)}}\right)-\frac{\bar{V}}{V_{0}}\right), \forall t \in N_{T} \\
\left(\omega_{t}^{-}\right)^{(k+1)}=\left(\omega_{t}^{-}\right)^{(k)}+\alpha\left(-1+\frac{1}{V_{0}^{2}} \operatorname{Re}\left(Z \overline{s_{t}^{(k)}}\right)+\frac{V}{V_{0}}\right), \forall t \in N_{T},
\end{gathered}
$$

where $s_{t}{ }^{(k)}$ is from the results of the solution of the inner problem and (11)-(12). Notice that the condition $\left(\lambda_{t}^{+}, \lambda_{t}^{-}, \omega_{t}^{+}, \omega_{t}^{-}\right) \geq 0$ should be checked; the violated ones are replaced by zeros.

Fourthly, the DTS is updated by the DSO using,

$$
\begin{gathered}
r_{t}^{(k+1)}=D^{T}\left(\left(\lambda_{t}^{+}\right)^{(k+1)}-\left(\lambda_{t}^{-}\right)^{(k+1)}\right)- \\
\frac{\operatorname{Re}\left(Z^{T}\right)}{V_{0}^{2}}\left(\left(\omega_{t}^{+}\right)^{(k+1)}-\left(\omega_{t}^{-}\right)^{(k+1)}\right) .
\end{gathered}
$$

At last, the convergence is checked, i.e., whether the subgradients tend to zero. If it is converged, the process stops and the DTSs determined in the fourth step are final. Otherwise, go back to the second step.

In fact, the above procedure is consistent with the procedure illustrated in Fig. 2. It should be noted that, in the third step, when the aggregators report their solutions to the DSO, they do not have to report a detailed solution, but the aggregated demands at each bus. As such, they can protect the privacy of their own businesses.

\section{CASE STUDIES}

\section{A. Case study parameters}

The four-feeder distribution network of the Roy Billinton Test System (RBTS) [32] is used for the case studies and the single line diagram of the system is shown in Fig. 3. Line segments of the first feeder are labeled in Fig. 3, among which L2, L4, L6, L8, L9, L11, and L12 refer to the transformers connecting the corresponding load points (LP1 to LP7). The study is focused on this feeder because it has the most diversity among all the feeders: 5 residential load points with different peak conventional demands and two commercial load points. The detailed data of these load points are listed in Ta- ble II. Line parameters are listed in Table III. The peak conventional demands of residential customers are assumed to occur at 18:00 when people arrive home and start cooking. Assume that the EVs and HPs have unit power factor. The DSO has improved the power factor of the conventional consumption by reactive power compensation, and the remaining reactive power consumption is $10 \%$ of the conventional active power consumption.

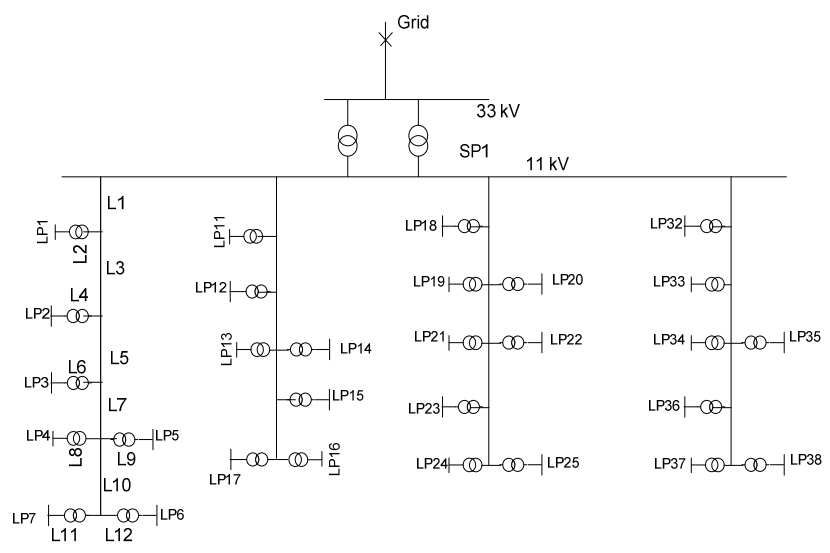

Fig. 3. Single line diagram of the distribution network

The key parameters of the simulation are listed in Table IV. The EV availability shown in Fig. 4 is from the driving pattern study in [33]. The heat demand of each household depends on its size. In this study, the household size is chosen randomly in the range between 100 and $200\left(\mathrm{~m}^{2}\right)$. Assume that there are 200 households per residential load points (LP1-5). Each household has one EV, one HP and one PV. A typical day with peak power $10 \mathrm{~kW}$ per PV is chosen for the study. The 24-hour production profile follows the data from Energy Data [34] provided by the Danish TSO, 'Energinet'.

Two aggregators are considered in this study. One has contracts with 160 households per bus, while the other has contracts with the rest 40 households per bus.

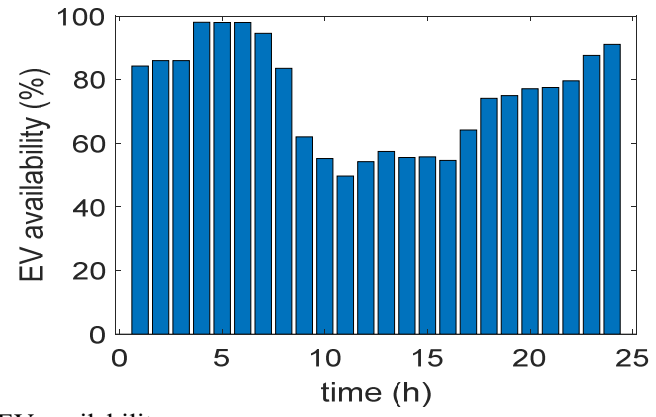

Fig. 4. EV availability

TABLE II

LOAD POINT DATA

\begin{tabular}{lllll}
\hline $\begin{array}{c}\text { load } \\
\text { points }\end{array}$ & $\begin{array}{c}\text { customer } \\
\text { type }\end{array}$ & $\begin{array}{c}\text { peak conv. } \\
\text { act. power } \\
\text { per point } \\
\text { (kW) }\end{array}$ & $\begin{array}{c}\text { peak conv. } \\
\text { react. power } \\
\text { per point } \\
\text { (kvar) }\end{array}$ & $\begin{array}{c}\text { number of } \\
\text { customers } \\
\text { per point }\end{array}$ \\
\hline LP1-LP4 & residential & 886.9 & 88.69 & 200 \\
\hline LP5 & residential & 813.7 & 81.37 & 200 \\
\hline LP6, LP7 & commercial & 671.4 & 67.14 & 10 \\
\hline
\end{tabular}


TABLE III

LINE PARAMETERS

\begin{tabular}{llll}
\hline Line/Trans. & $\mathbf{r}(\mathbf{o h m})$ & $\mathbf{x}(\mathbf{o h m})$ & $\mathbf{x} / \mathbf{r}$ ratio \\
\hline 1 & 0.1210 & 0.0370 & 0.3058 \\
\hline 2 & 0.3000 & 3.0000 & 10.0000 \\
\hline 3 & 0.4233 & 0.0943 & 0.2228 \\
\hline 4 & 0.3000 & 3.0000 & 10.0000 \\
\hline 5 & 0.3722 & 0.0829 & 0.2227 \\
\hline 6 & 0.3000 & 3.0000 & 10.0000 \\
\hline 7 & 0.4403 & 0.0981 & 0.2228 \\
\hline 9 & 0.3000 & 3.0000 & 10.0000 \\
\hline 10 & 0.3000 & 3.0000 & 10.0000 \\
\hline 11 & 0.3091 & 0.0689 & 0.2229 \\
\hline 12 & 0.3000 & 3.0000 & 10.0000 \\
\hline
\end{tabular}

TABLE IV

Key Parameters of the Simulation Model ([33], [35])

\begin{tabular}{ll}
\hline \multicolumn{1}{c}{ parameter } & \multicolumn{1}{c}{ value } \\
\hline EV battery size & $25 \mathrm{kWh}$ \\
\hline Peak charging power & $11 \mathrm{~kW}(3$ phase $)$ \\
\hline Energy consumption per km & $150 \mathrm{Wh} / \mathrm{km}$ \\
\hline Minimum SOC & $10 \%$ \\
\hline Maximum SOC & $90 \%$ \\
\hline Average driving distance & $40 \mathrm{~km}$ \\
\hline Coefficient of performance (COP) of HP & 2.3 \\
\hline Min Temp. of the House & $20^{\circ} \mathrm{C}$ \\
\hline Max Temp. of the House & $24{ }^{\circ} \mathrm{C}$ \\
\hline Voltage rating $=V_{0}$ & $11 \mathrm{kV}=1 \mathrm{p} . \mathrm{u}$. \\
\hline Lower/upper voltage limit & $0.94 / 1.06 \mathrm{p.u}$. \\
\hline L2-4 limit $(\mathrm{kW})$ & $1100 / 7000 / 2700$ \\
\hline Power tariff $(\mathrm{DKK} / \mathrm{KWH} / \mathrm{KWH})$ & 0.01 \\
\hline
\end{tabular}

\section{B. Case study results}

For brevity, only the dual decomposition method for DTS calculation is simulated in this study. The simulation was carried out using the GAMS optimization software [36] for the aggregator optimization, and a Matlab script for the iteration control, convergence check and the DSO side DTS calculation. Interface between GAMS and Matlab for communicating DTS signal and DR results is through 'GAMS Data eXchange' (GDX).

1) Congestion Management Results:

Initially, the DTSs are zeros. The aggregators perform the optimal scheduling with zero DTSs. The results are reported to the DSO, who then put the results together to have the overall load flow profile. For privacy concerns, the aggregators do not report planning of EV, HP and PV separately. But here, in order to see the efficacy of the DTS method for DER management, line flows of EV, HP and PV are plotted separately first. Then the total line flow profiles are plotted, as shown in Fig. 5. For brevity, only line flows of L2-4 are plotted. Voltage profiles are not plotted because voltage congestion does not occur in this study.

From the results, it can be seen that in addition to the morning and evening peaks/congestion, the day-time feed-in power congestion occurs due to too much PV power. The V2G function is also observed in several hours, especially in hour 1, 2, 14 and 16 due to relatively high forecast day-ahead energy prices (see the base price in Fig. 6). The day-ahead energy prices are largely influenced by wind and other renewable energy forecast. EVs are expected to charge at lower prices and discharge (V2G) at higher prices to make profits. Without coordination (DTS $=0$ ), DERs can cause severe congestion problems to the distribution networks as in this case.
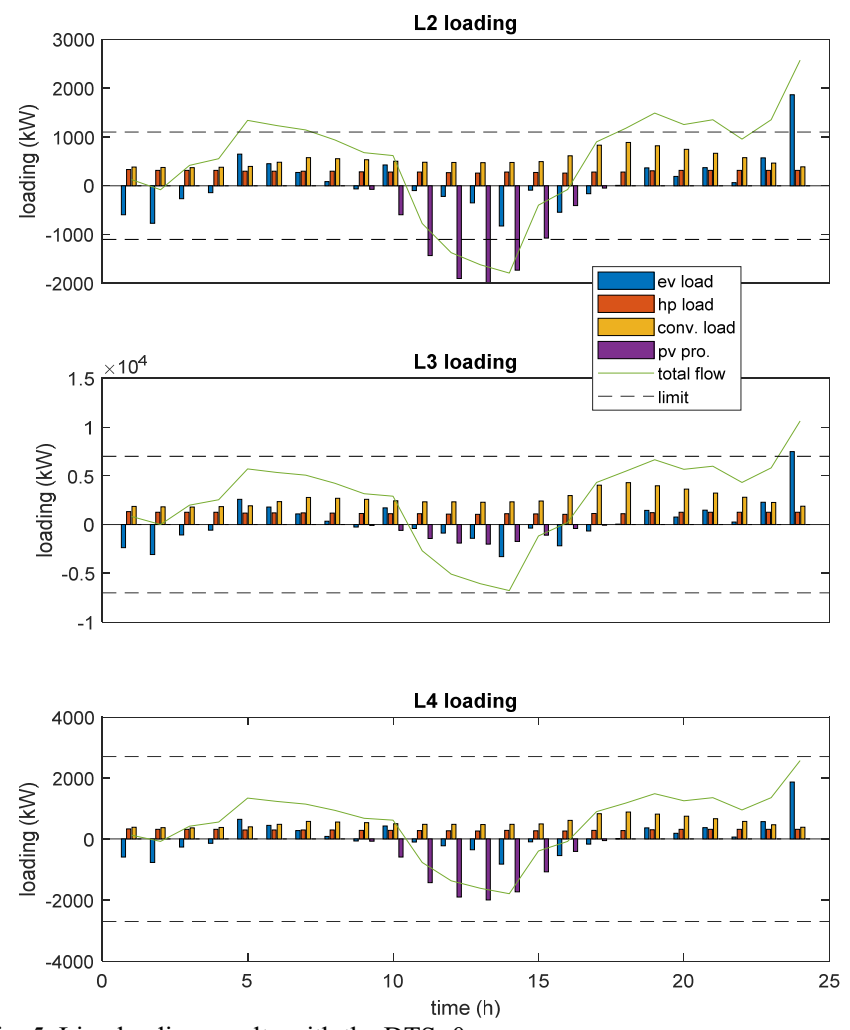

Fig. 5 . Line loading results with the DTS $=0$

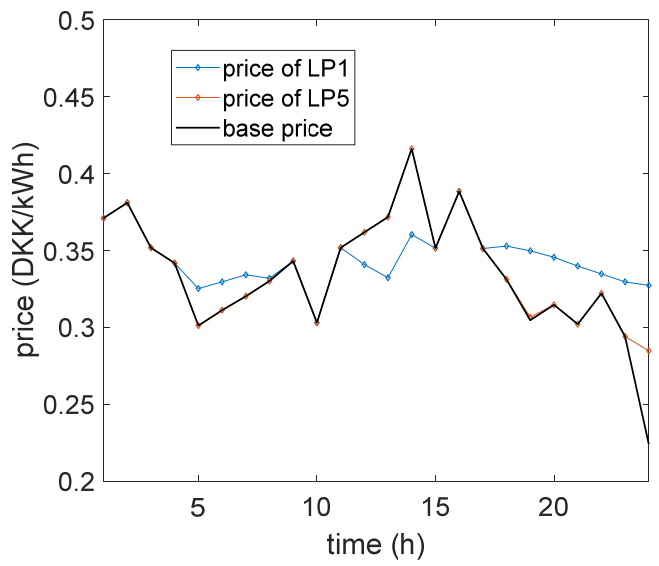

Fig. 6. Forecast system prices (base price) and final prices (containing DTS)

Then the iteration starts and the information is exchanged between the DSO and aggregators. Finally, the congestion due to DER is resolved and the results are shown in Fig. 6 and Fig. 7.

For downward power flow (from the external grid to the distribution network) congestion, it can be resolved by increasing the DTS rates. When the forecast energy price plus the DTS is high, EVs and HPs are expected to reduce power consumption and the congestion is resolved. Also, V2G is encouraged due to high prices and it helps congestion mitigation. This is the case for morning and evening peak hour congestion. DTSs are increased in hour 5- 7 and 18-24, and the EVs and HPs reduce their consumption due to high DTSs. 
On the other hand, for upward power flow congestion due to PV and V2G, it can be resolved by reducing DTS rates, including negative rates. This is the case for hour 12-14. Because of heavy PV production, DTS rates become negative in order to increase EV and HP consumption and reduce V2G power. It can be concluded that the DTS method has successfully coordinated the DERs in the distribution network and the congestion has been resolved in both directions.

\section{2) Convergence Observation:}

The key variables, including the line flow (L2, transformer), corresponding marginal prices $\lambda_{t}^{+}, \lambda_{t}^{-}$, and DTS rates (LP1) are observed in the iteration process. The results are shown in Fig. 8. It can be seen that the iteration process is quite stable, indicating a stable algorithm of the dual decomposition. The process is settled after about 150 iterations (further observation thereafter is for the study purpose). The marginal prices $\lambda_{t}^{-}$of L2 are positive for hour 14 and zero for other hours (the second subplot of Fig. 8).

The computational complexity is low since the dual decomposition method is employed. Each decomposed problem (down to each EV or HP) is quite small and can be solved in much less than one second. The bottleneck is the communication time. When implementing the DTS method in a real-life application, the iteration process and the communication between the DSO and aggregators should be automated. The communication time for each iteration should be kept within 1 or 2 seconds.
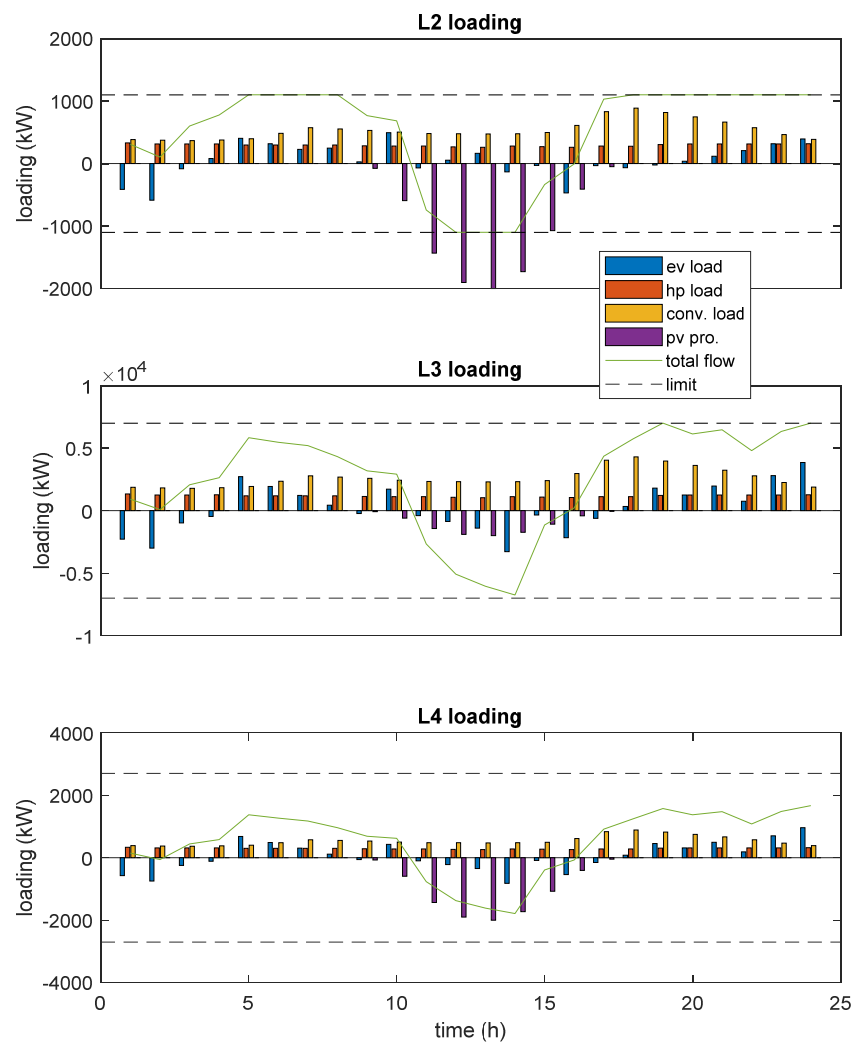

Fig. 7. Line loading results after using the DTS method
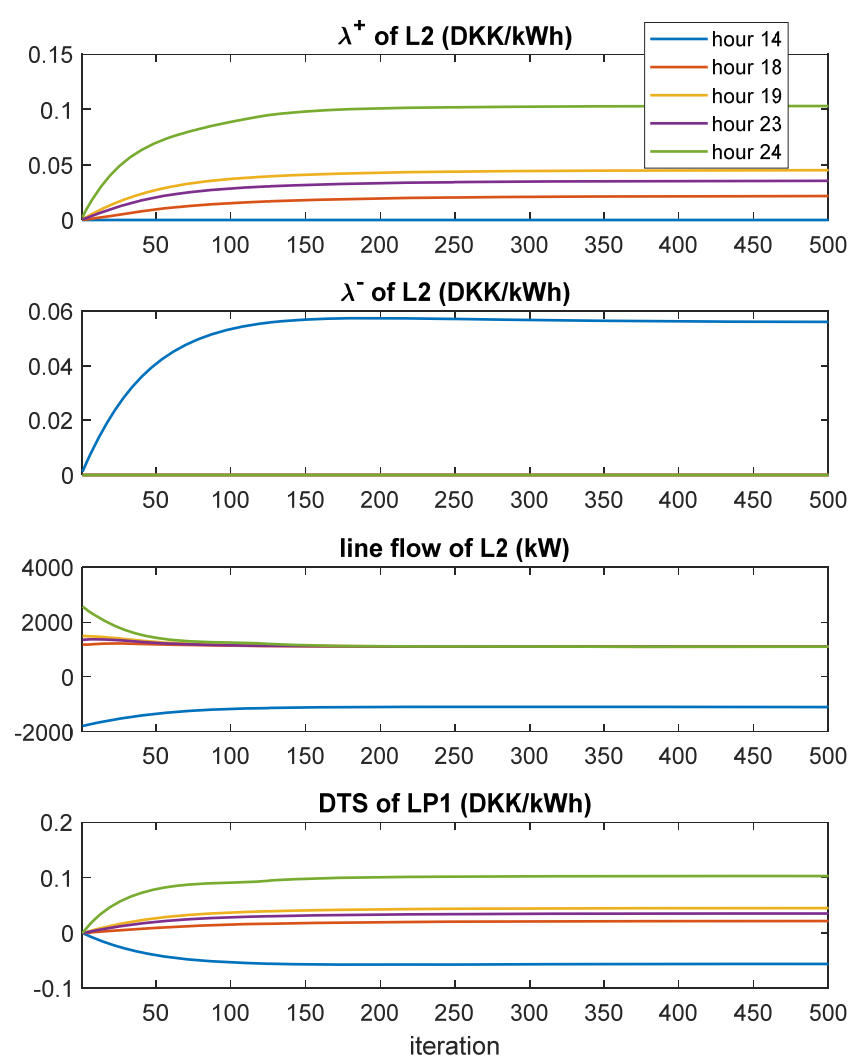

Fig. 8 Iteration observation of the key variables

\section{CONCLUSIONS}

This paper proposes the DTS method for congestion management in distribution networks with high penetration of PVs, HPs and EVs with V2G function. The DTS method is a very important extension of the DT method. With this extension, the regulation prices can be negative, which can effectively solve the congestion problems due to feed-in power flows. Also, the extension allows EVs to work at both G2V and $\mathrm{V} 2 \mathrm{G}$ modes. Therefore, the DTS method can be employed for efficient congestion management of all kinds of DERs, as PV, HP and EV with V2G can represent almost all types of DERs in distribution networks.

\section{APPENDIX}

\section{A. DT Concept}

A brief description of the DT is explained here while more details can be found in [7]. The procedure of using the DT concept to solve the congestion problem in a decentralized manner can be summarized as follows. Firstly, the DSO obtains the flexible demand data, such as energy requirements and the availability, from the aggregators or by its own prediction. The DSO also needs the network information and the predicted spot price. Secondly, the DTs are calculated through the optimal plan respecting the network constraints, and the DTs are published to all the aggregators. Thirdly, after receiving the DTs, the aggregators make their own optimal plans independently with both the predicted spot prices and the DTs. At last, the aggregators submit their energy plan/bids to the spot market. 


\section{B. The B Matrix and Quadratic Programming}

As proven in [7], quadratic programming can solve the 'multiple-solution' problem that may appear in linear programming formulation of the DT method. Multiple-solution means that the aggregators have many possible optimal solutions for a given price set (forecast energy price + DT), leading to a failure of the congestion management since many of the optimal solutions do not respect the network constraint. Linear programming can also have the converge problems if multiple-solution appears for the case of dual decomposition.

A proper cause of the quadratic terms is either as suggested in [7] using the concept of price sensitivities (the final energy price is the forecast price + a sensitive part which is proportional to the planed power of the flexible demands), or as in this paper using the power tariff concept. The $B$ matrix is a diagonal matrix, whose elements are identical power tariffs in this paper.

\section{HP Modelling}

In this subsection, the HP modelling is presented. The full version of the derivation can be found in [7]. The heat transfer process of the air source HP can be represented by an electric circuit [37] which is illustrated in Fig. 9. Thus, the following thermal balance equations can be derived [37].

$$
\begin{gathered}
Q_{t}^{e}+S_{t}^{1}-k_{1}\left(K_{t}^{a}-K_{t}\right)-k_{2}\left(K_{t}^{a}-K_{t}^{s}\right)= \\
C_{a}\left(K_{t}^{a}-K_{t-1}^{a}\right), \forall \mathrm{t} \in N_{T} \\
S_{t}^{2}+k_{2}\left(K_{t}^{a}-K_{t}^{s}\right)-k_{3}\left(K_{t}^{s}-K_{t}\right)= \\
C_{s}\left(K_{t}^{s}-K_{t-1}^{s}\right), \forall \mathrm{t} \in N_{T}
\end{gathered}
$$

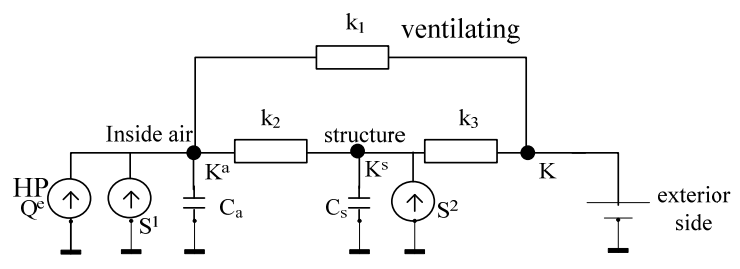

Fig. 9. Heat transferring process of the house

Equations (26) and (27) can be solved iteratively. As a result, the house inside air temperature $K_{t}^{a}$ will be a linear combination of all the previous and current thermal energy $\left(Q_{t}^{e}\right)$ plus the initial state. Because $Q_{t}^{e}$ has a linear relation (by the coefficient of performance (COP)) to the active power $\hat{p}_{t}$ consumed by the HP, the house inside air temperature can be expressed as,

$$
K_{t}^{a}=\sum_{t_{-} \leq t} a_{t, t_{-}} \hat{p}_{t_{-}}+u_{t} \quad \forall \mathrm{t} \in N_{T} .
$$

Finally, the matrix form for (28) is written as,

$$
K_{i, t}^{a, \min } \leq \sum_{t_{-} \leq t} A_{i, t, t_{-}} \hat{p}_{i, t_{-}}+u_{i, t} \leq K_{i, t}^{a, \max }, \forall i \in \mathrm{N}_{B}, \mathrm{t} \in N_{T}
$$

where, $A_{i, t, t}$ is a diagonal matrix and $u_{i, t}$ is a constant vector, obtained by combining the varying part (power related) and the constant part of (26) - (27), respectively.

\section{REFERENCES}

[1] S. Huang, Q. Wu, Z. Liu, and A. H. Nielsen,"Review of congestion management methods for distribution networks with high penetration of distributed energy resources," in Proc. IEEE PES Innovative Smart Grid Technologies, Europe, pp. 1-6.

[2] J. F. Franco, M. J. Rider, M. Lavorato, and R. Romero,"A mixed-integer LP model for the reconfiguration of radial electric distribution systems considering distributed generation," Electr. Power Syst. Res., vol.97, pp. 51-60, Apr. 2013.

[3] S. Balaraman and N. Kamaraj, "Application of differential evolution for congestion management in power system," Mod. Appl. Sci., vol.4, no.8, pp. 33-42, Jul. 2010.

[4] K. Daroj and W. Limpananwadi,"Reactive power dispatch scheme evaluation for synchronous based distributed generators to reduce real power loss in distribution systems," in Proc. IEEE International Conference on Sustainable Energy Technologies, pp. 1178-1183.

[5] N. O'Connell, Q. Wu, J. Østergaard, A. H. Nielsen, S. T. Cha, and Y. Ding,"Day-ahead tariffs for the alleviation of distribution grid congestion from electric vehicles," Electr. Power Syst. Res., vol.92, pp. 106-114, 2012.

[6] R. Li, Q. Wu, and S. S. Oren,"Distribution locational marginal pricing for optimal electric vehicle charging management," IEEE Trans. Power Syst., vol.29, no.1, pp. 203-211, Jan. 2014.

[7] S. Huang, Q. Wu, S. S. Oren, R. Li, and Z. Liu,"Distribution locational marginal pricing through quadratic programming for congestion management in distribution networks," IEEE Trans. Power Syst., vol.30, no.4, pp. 2170-2178, Jul. 2015.

[8] S. Huang, Q. Wu, L. Cheng, and Z. Liu, “Optimal reconfiguration-based dynamic tariff for congestion management and line loss reduction in distribution networks," IEEE Trans. Smart Grid, vol.7, no.3, pp. 12951303, May 2016.

[9] P. M. Sotkiewicz and J. M. Vignolo,"Nodal pricing for distribution networks: Efficient pricing for efficiency enhancing DG," IEEE Trans. Power Syst., vol.21, no.2, pp. 1013-1014, May 2006.

[10] G. T. Heydt, B. H. Chowdhury, M. L. Crow, D. Haughton, B. D. Kiefer, F. Meng, and B. R. Sathyanarayana,"Pricing and control in the next generation power distribution system," IEEE Trans. Smart Grid, vol.3, no.2, pp. 907-914, Jun. 2012.

[11] B. Biegel, P. Andersen, J. Stoustrup, and J. Bendtsen,"Congestion management in a smart grid via shadow prices," in Proc. 2012 8th IFAC Symposium on Power Plant and Power System Control, pp. 518-523.

[12] M. R. Sarker, M. A. Ortega-Vazquez, and D. S. Kirschen, "Optimal coordination and scheduling of demand response via monetary incentives," IEEE Trans. Smart Grid, vol.6, no.3, pp. 1341-1352, May 2015.

[13] H. Zhong, L. Xie, and Q. Xia,"Coupon incentive-based demand response: theory and case study," IEEE Trans. Power Syst., vol.28, no.2, pp. 1266-1276, May 2013.

[14] C. Zhang, Y. Ding, N. Nordentoft, P. Pinson, and J. Østergaard,"FLECH-A danish market solution for DSO congestion management through DER flexibility services," J. Mod. Power Syst. Clean Energy, vol.2, no.2, pp. 126-133, 2014.

[15] S. Huang and Q. Wu,"Dynamic Subsidy Method for Congestion Management in Distribution Networks," IEEE Trans. Smart Grid, vol.9, no.3, pp. 2140-2151, May 2018.

[16] B. Moradzadeh and K. Tomsovic,"Two-stage residential energy management considering network operational constraints," IEEE Trans. Smart Grid, vol.4, no.4, pp. 2339-2346, Dec. 2013.

[17] S. Weckx, R. D'Hulst, B. Claessens, and J. Driesensam,"Multiagent charging of electric vehicles respecting distribution transformer loading and voltage limits," IEEE Trans. Smart Grid, vol.5, no.6, pp. 28572867, Nov. 2014.

[18] L. Gan, U. Topcu, and S. H. Low, "Optimal decentralized protocol for electric vehicle charging," IEEE Trans. Power Syst., vol.28, no.2, pp. 940-951, May 2013.

[19] Z. Fan,"A distributed demand response algorithm and its application to PHEV charging in smart grids," IEEE Trans. Smart Grid, vol.3, no.3, pp. 1280-1290, Sep. 2012.

[20] M. Esmaili, N. Amjady, and H. A. Shayanfar,"Stochastic congestion management in power markets using efficient scenario approaches," Energy Convers. Manag., vol.51, no.11, pp. 2285-2293, 2010.

[21] M. Hojjat and M. H. Javidi D. B.,"Probabilistic congestion management considering power system uncertainties using chance-constrained 
programming," Electr. Power Components Syst., vol.41, no.10, pp. 972 989, Jul. 2013.

[22] Z. Liu, Q. Wu, S. Oren, S. Huang, R. Li, and L. Cheng,"Distribution locational marginal pricing for optimal electric vehicle charging through chance constrained mixed-integer programming," IEEE Trans. Smart Grid, vol. 9, no. 2, pp. 644-654, Mar. 2018.

[23] S. Huang, Q. Wu, L. Cheng, Z. Liu, and H. Zhao,"Uncertainty management of dynamic tariff method for congestion management in distribution networks," IEEE Trans. Power Syst., vol.31, no.6, pp. 43404347, Nov. 2016.

[24] G. R. C. Mouli, M. Kefayati, R. Baldick, and P. Bauer,"Integrated PV Charging of EV Fleet Based on Energy Prices, V2G and Offer of Reserves," IEEE Trans. Smart Grid, in press.

[25] J. Romero-Ruiz, J. Pérez-Ruiz, S. Martin, J. A. Aguado, and S. De la Torre,"Probabilistic congestion management using EVs in a smart grid with intermittent renewable generation," Electr. Power Syst. Res., vol.137, pp. 155-162, Aug. 2016.

[26] M. Ghofrani, A. Arabali, and M. Ghayekhloo,"Optimal charging/discharging of grid-enabled electric vehicles for predictability enhancement of PV generation," Electr. Power Syst. Res., vol.117, pp. 134-142, Dec. 2014.

[27] S. Huang, Q. Wu, M. Shahidehpour, and Z. Liu,"Dynamic Power Tariff for Congestion Management in Distribution Networks," IEEE Trans. Smart Grid, in press.

[28] S. Huang, Q. Wu, H. Zhao, and C. Li,"Distributed Optimization based Dynamic Tariff for Congestion Management in Distribution Networks," IEEE Transactions on Smart Grid, in press.

[29] S. Bolognani and S. Zampieri,"On the existence and linear approximation of the power flow solution in power distribution networks," IEEE Trans. Power Syst., vol.31, no.1, pp. 163-172, Jan. 2016.

[30] S. Boyd, L. Xiao, A. Mutapcic, and J. Mattingley, "Notes on decomposition methods," $2008 . \quad$ Available: https://pdfs.semanticscholar.org/d746/f6b5f69401b147996d21a4b5940f 6dd40020.pdf. [Accessed: 26-Mar-2017].

[31] S. Boyd, L. Xiao, and A. Mutapcic,"Subgradient Methods," 2003. Available: https://web.stanford.edu/class/ee392o/subgrad_method.pdf. [Accessed: 27-Jun-2017].

[32] R. N. Allan, R. Billinton, I. Sjarief, L. Goel, and K. S. So,"A reliability test system for educational purposes-basic distribution system data and results," IEEE Trans. Power Syst., vol.6, no.2, pp. 813-820, May 1991.

[33] Q. Wu, A. H. Nielsen, J. Østergaard, F. Marra, and C. Træholt,"Driving pattern analysis for electric vehicle (EV) grid integration study," in Proc. 2010 IEEE PES Innovative Smart Grid Technologies Conference Europe (ISGT Europe), pp. 1-6.

[34] Energinet,"Energy data." Available: https://www.energidataservice.dk/en/. [Accessed: 16-Feb-2018].

[35] J. M. Nyers and A. J. Nyers, "COP of heating-cooling system with heat pump," in Proc. IEEE 3rd International Symposium on Exploitation of Renewable Energy Sources (EXPRES), pp. 17-21.

[36] R. E. Rosenthal. (2014, Aug. ). GAMS - A user's guide. GAMS Development Corporation, Washington, DC, USA. [online]. Available: http://www.gams.com/dd/docs/bigdocs/GAMSUsersGuide.pdf.

[37] P. Bacher and H. Madsen, "Identifying suitable models for the heat dynamics of buildings," Energy Build., vol.43, no.7, pp. 1511-1522, Jul. 2011.

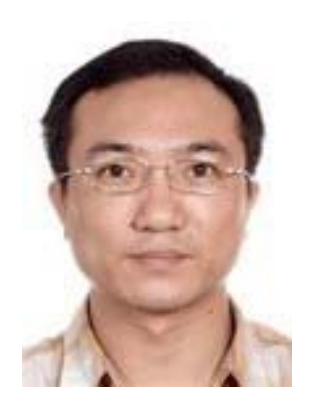

IX. BIOGRAPHIES

Shaojun Huang (M'13, SM'18) obtained the B.Eng. from the Department of Electrical Engineering, Tsinghua University, Beijing, China, in 2001. He obtained the MSc from the Department of Energy Technology, Aalborg University, Denmark, in 2013. He obtained the $\mathrm{PhD}$ from the Department of Electrical Engineering, Technical University of Denmark, Denmark, in 2016. Currently, he is an assistant professor at the Centre for Energy Informatics, MMM Institute, University of Southern Denmark. His research interests include renewable energy, demand-side management, smart grid, and system optimization.

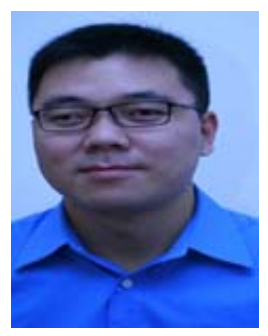

Qiuwei Wu (M'08-SM'15) obtained obtained the $\mathrm{PhD}$ degree in Power System Engineering from Nanyang Technological University, Singapore, in 2009. He was a senior R\&D engineer with VESTAS Technology R\&D Singapore Pte Ltd from Mar. 2008 to Oct. 2009. He is an Associate Professor at Department of Electrical Engineering, Technical University of Denmark (DTU) since Nov. 2009. He was a visiting scholar at Department of Industrial Engineering \& Operations Research (IEOR), University of California, Berkeley, from Feb. 2012 to May 2012 funded by Danish Agency for Science, Technology and Innovation (DASTI), Denmark. He was a visiting scholar at School of Engineering and Applied Sciences, Harvard University from Nov. 2017 to Oct. 2018.

His research area is power system operation and control with high renewables, including wind power modelling and control, active distribution networks, and integrated energy systems. He is an Editor of IEEE Transactions on Smart Grid and IEEE Power Engineering Letters. He is also an Associate Editor of International Journal of Electrical Power and Energy Systems, Journal of Modern Power Systems and Clean Energy, IET Renewable Power Generation, and IET Generation, Transmission \& Distribution. 